\title{
Comparison of incremental and constant load tests of inspiratory muscle endurance in COPD
}

\author{
K. Hill*,\#,ף, S.C. Jenkins ${ }^{\#,{ }^{*},+}$, D.L. Philippe*, K.L. Shepherd*, \\ D.R. Hillman* and P.R. Eastwood*,ף,ऽ
}

ABSTRACT: The aim of the present study was to determine the relative value of incremental and constant load tests in detecting changes in inspiratory muscle endurance following high-intensity inspiratory muscle training (H-IMT) in chronic obstructive pulmonary disease.

In total, 16 subjects (11 males; forced expiratory volume in one second (FEV1) $37.4 \pm 12.5 \%$ ) underwent H-IMT. In addition, 17 subjects (11 males; FEV $136.5 \pm 11.5 \%$ ) underwent sham inspiratory muscle training (S-IMT). Training took place three times a week for 8 weeks. Baseline and post-training measurements were obtained of maximum threshold pressure sustained during an incremental load test ( $P_{\text {th,max }}$ ) and time breathing against a constant load (tlim). Breathing pattern was unconstrained.

H-IMT increased $P$ th,max and $t$ lim relative to baseline and to any change seen following S-IMT. The effect size for $P$ th,max was greater than for $t$ lim. Post-training tests were accompanied by changes in breathing pattern, including decreased duty cycle, which may have served to decrease inspiratory work and thereby contribute to the increase in $P_{\text {th, }}$ max and $t$ lim in both groups.

When assessing inspiratory muscle function in chronic obstructive pulmonary disease via tests in which the pattern of breathing is unconstrained, the current authors recommend incremental load tests be used in preference to constant load tests. However, to attribute changes in these tests to improvements in inspiratory muscle endurance, breathing pattern should be controlled.

KEYWORDS: Assessment, endurance, lung disease, respiratory muscles

I nspiratory muscle function is most often described in terms of the maximum force generating capacity (i.e. strength) and the capacity to maintain a specific muscular task over time (i.e. endurance) [1]. The measurement of maximum inspiratory pressure $(P \mathrm{I}, \mathrm{max})$ is widely accepted and commonly used as a simple assessment of global inspiratory muscle strength $[1,2]$. However, the physiological relevance of $P \mathrm{I}, \mathrm{max}$ is unclear given that maximal inspiratory pressures are rarely generated during activities of daily living in either healthy individuals or those with respiratory disease. As the inspiratory muscles are required to perform submaximal contractions throughout life, it is possible that assessment of their endurance may be of greater functional relevance than measurements of their strength. In contrast to the measurement of PI,max, no such generally accepted method exists for the measurement of global inspiratory muscle endurance [1, 3], although both the maximum threshold pressure sustained during an incremental load test $\left(P_{\text {th,max }}\right)$ and the time to exhaustion while inspiring against a constant submaximal load (tlim) are commonly used for this purpose $[2,4,5]$.

Following inspiratory muscle training (IMT) many studies have reported improvements in the performance of incremental or constant load tests of inspiratory muscle endurance relative to baseline values [6-15]. However, reports of such improvements are less common when changes are compared with those observed in a control group [9,11-13]. This disparity suggests that these tests may have limited specificity for the detection of training responses to IMT programmes.

In a recent analysis of the effect of an 8-week programme of high-intensity IMT on inspiratory muscle function, the current authors demonstrated a $56 \%$ increase in endurance as assessed by incremental loading $\left(P_{\text {th, } \max }\right)$ [16]. In the present study, this analysis has been extended to compare this measure of endurance with the
AFFILIATIONS

Depts of *Pulmonary Physiology, and \#Physiotherapy, Sir Charles Gairdner Hospital,

+Lung Institute of Western Australia, ${ }^{\text {s }}$ School of Anatomy and Human Biology, University of Western Australia, Nedlands, and "School of Physiotherapy, Curtin University of Australia, Bentley, Perth, Western Australia, Australia.

CORRESPONDENCE

P.R. Eastwood

Dept of Pulmonary Physiology

Sir Charles Gairdner Hospital

Hospital Avenue

Nedlands

Western Australia

Australia 6009

Fax: 61893462034

E-mail: peter.eastwood@

health.wa.gov.au

Received:

July 202006

Accepted after revision:

April 282007

SUPPORT STATEMENT

This study was supported by the National Health and Medical Research Council (Canberra, Australia) grant number 212016.

STATEMENT OF INTEREST

None declared.

European Respiratory Journal Print ISSN 0903-1936 Online ISSN 1399-3003 
assessment of endurance by a constant load test. The purpose of doing so was to determine: 1) their value in detecting changes in muscle performance relative to baseline values; and 2) the capacity of each to discriminate between a treatment and control group. The results of such analyses could be helpful in determining which type of test is of greater value in investigating respiratory muscle endurance and response to training in clinical and research settings.

\section{METHODS}

\section{Subjects}

The subjects recruited for the present study have been described in detail elsewhere [16]. Briefly, subjects were recruited who had a forced expiratory volume in one second (FEV1) of $15-70 \%$ of the predicted normal value [17], a medical diagnosis of chronic obstructive pulmonary disease (COPD) and a smoking history $>10$ pack-yrs. Exclusion criteria were the use of long-term oxygen therapy, tapering doses of corticosteroids or methylxanthines, a history of lung surgery or spontaneous pneumothorax, and any comorbid condition thought to adversely affect test performance.

\section{Study design}

A prospective double-blind randomised controlled trial was undertaken to compare the effects of high-intensity, intervalbased IMT (H-IMT) with sham IMT (S-IMT). All subjects entered a 2-week "screening and familiarisation" phase, during which time all baseline measurements were collected. To account for any improvements due to familiarisation with the tests [18], both incremental and constant load tests of inspiratory muscle endurance were performed on four separate occasions ( $>24 \mathrm{~h}$ apart) prior to commencing training. Subjects were then randomised to receive 8 weeks of H-IMT or S-IMT. On completion of the training period, measurements were repeated. Specific details of the measurements of resting lung function, inspiratory muscle strength, exercise capacity, dyspnoea and health-related quality of life and the changes in these measurements following H-IMT and S-IMT have been described previously [16]. Approval for the study was obtained from the Human Research Ethics Committees of Sir Charles Gairdner Hospital (Nedlands, Australia) and Curtin University of Technology (Bentley, Australia) and written informed consent was obtained from each subject prior to participation.

\section{Measurements}

Inspiratory muscle endurance

Inspiratory muscle endurance was assessed using an incremental [4] and a constant load test [5]. Details of the assessment procedures have been described elsewhere [16]. Briefly, subjects were seated, wore a nose-clip and received standardised instructions and encouragement to facilitate maximal performance. Both tests were performed using a modified threshold loading valve $[19,20]$ and measurements of inspiratory mouth pressure (differential pressure transducer; Honeywell, Morristown, NJ, USA), inspiratory flow and tidal volume (Fleisch no. 2 pneumotachograph and differential pressure transducer; Validyne Engineering, Northridge, CA, USA) were continuously recorded on an electronic strip chart recording system (PowerLab/16s; ADInstruments Pty Ltd, Castle Hill, Australia). Subjects were permitted to choose their own breathing pattern throughout both tests. Inspiratory time was defined as the time period extending from the commencement of an increase in inspiratory pressure to the end of inspiratory flow [21]. Expiratory time was defined as the remainder of the total respiratory cycle time [21]. The pressure-time index (PTI) was calculated as the product of inspiratory duty cycle and the proportion of PI,max generated with each inspiratory effort $[1,22,23]$.

Incremental load test

The incremental load test required subjects to breathe against inspiratory threshold loads that were increased each minute by $10 \%$ of baseline $P \mathrm{I}$,max until voluntary task failure. Inspiratory muscle endurance was defined as the $P$ th,max sustained for a minimum of $30 \mathrm{~s}$ [18]. The highest $P$ th, $\max$ achieved during the "screening and familiarisation" phase was recorded as the baseline measure. The load increments were kept identical between baseline and post-training tests. Breathing pattern variables were averaged over the final 20-s epoch of the maximum load achieved $\left(P_{\text {th, }}\right.$ max $)$ during the baseline and post-training tests. Any breaths affected by artefact (e.g. swallowing) were excluded from the analyses.

\section{Constant load test}

The constant load test was performed $\geqslant 15 \mathrm{~min}$ after completion of the incremental load test. Subjects were required to breathe against a submaximal threshold load until task failure, with inspiratory muscle endurance defined as the time able to breathe against the load (tlim). An inspiratory load was selected such that tlim was 5-10 min [24]. Determination of this load was undertaken during the "screening and familiarisation" phase by increasing inspiratory loads if tlim was $>15 \mathrm{~min}$ and reducing them if tlim was $<1 \mathrm{~min}$. Once determined, this load was used during all pre- and post-training assessments. While the absolute magnitude of the load varied between individuals, it was equivalent to $\sim 80 \%$ of baseline $P$ th,max in all subjects, being of similar magnitude to that used by RAMIREZSARMIENTO et al. [14] in a similar group of subjects. The longest tlim achieved against this load during the "screening and familiarisation" phase was selected as the baseline value. In the event that a subject sustained inspiratory efforts against the constant load for $15 \mathrm{~min}$ on any test occasion, the investigator terminated the test and recorded the thim as $15 \mathrm{~min}$. Breathing pattern variables were averaged over the final minute of each of the baseline and post-training tests. Any breaths affected by artefact (e.g. swallowing) were excluded from these analyses.

\section{Inspiratory muscle training}

Training was supervised and took place three times a week for 8 weeks. A novel interval-based H-IMT programme using a modified threshold training device (Threshold IMT; Respironics, Cedar Grove, NJ, USA) was applied, which comprised seven cycles of 2 min loaded breathing followed by a 1-min rest [25]. To familiarise subjects with the handheld training device, a low inspiratory load $(45 \%$ of the pre-training PI,max) was applied during the first training session. The load was increased so that following the third session, subjects were generating inspiratory pressures equivalent to $\sim 60 \%$ of the pre-training PI,max with each breath. Subjects were permitted to choose their own breathing pattern. The load was further increased over the 8-week period with the aim of titrating to a 
level where subjects were just able to complete the final 2-min interval. Subjects allocated to receive S-IMT underwent training at a load equivalent to $10 \%$ of the previously determined PI,max, which was unchanged throughout the training programme. Further details of the H-IMT and S-IMT programmes have been described elsewhere [16].

\section{Data management and statistical analyses}

An $\alpha$ (p)-value $<0.05$ was considered significant. All data are expressed as mean $\pm \mathrm{SD}$, unless otherwise stated.

\section{Analyses}

Baseline measurements were compared between groups using unpaired t-tests (continuous variables) or Chi-squared tests (categorical variables). Data that deviated significantly from the normal distribution were transformed. Differences between and within groups were analysed using independent and paired t-tests, respectively.

Cohen's $d$ effect sizes were calculated by dividing the difference in the magnitude of change in each measure between the groups by the pooled SD of the mean change in each group. The capacity of each measure to discriminate individuals who had participated in H-IMT or S-IMT was evaluated in terms of their sensitivity and specificity, presented together as receiver operating characteristic (ROC) curves. The area under the curve for each ROC plot and the associated 95\% confidence interval were compared to assess the ability of each measure to discriminate between individuals in the H-IMT and S-IMT groups and, therefore, the effect of training.

\section{RESULTS}

\section{Subjects}

In total, 16 subjects (FEV1 $37.4 \pm 12.5 \%$ pred; age $69.4 \pm 7.2$ yrs; 11 males) completed the H-IMT programme and 17 subjects (FEV1 $36.5 \pm 11.5 \%$ pred; age $66.6 \pm 9.8 \mathrm{yrs} ; 11$ males) completed the S-IMT programme. Further details on subject characteristics are described elsewhere [16]. Prior to initiating

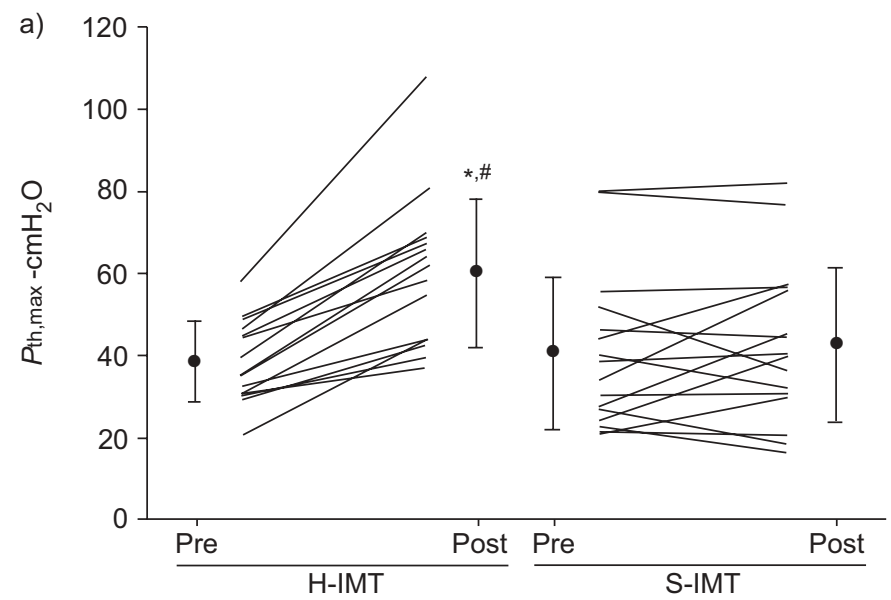

training, no significant differences were observed in any measure of resting lung function, inspiratory muscle strength or endurance, exercise capacity, dyspnoea or health-related quality of life between the H-IMT and S-IMT groups [16].

\section{Effects of H-IMT and S-IMT on measures of inspiratory muscle strength}

PI,max increased by $29 \%$ (from $6.57 \pm 1.61$ to $7.89 \pm 1.74 \mathrm{kPa}$ $\left(67.2 \pm 16.5\right.$ to $\left.\left.80.7 \pm 17.8 \mathrm{cmH}_{2} \mathrm{O}\right) ; \mathrm{p}<0.001\right)$ following H-IMT and by $8 \%$ (from $6.50 \pm 1.86$ to $7.01 \pm 1.83 \mathrm{kPa}(66.5 \pm 19.0$ to $\left.\left.71.7 \pm 18.7 \mathrm{cmH}_{2} \mathrm{O}\right) ; \mathrm{p}<0.05\right)$ following S-IMT [16]. The magnitude of the increase in PI,max was greater following H-IMT than the increase seen following S-IMT $(p=0.002)$ [16].

\section{Effects of H-IMT and S-IMT on measures of inspiratory muscle endurance}

Following H-IMT, Pth,max increased by $56 \%(2.11 \pm 1.06 \mathrm{kPa}$ $\left(21.6 \pm 10.8 \mathrm{cmH}_{2} \mathrm{O}\right) ; \mathrm{p}<0.001$; fig. 1a) and tim increased by $162 \% \quad(511 \pm 198 \mathrm{~s} ; \mathrm{p}<0.001$; fig. $2 \mathrm{a})$. Sixteen (100\%) subjects achieved a $P$ th,max greater than their baseline result, $15(94 \%)$ achieved a tlim greater than their baseline result and $12(75 \%)$ achieved a tlim of $15 \mathrm{~min}$.

Following S-IMT, Pth,max was unchanged (fig. 1a) and thim increased by $70 \%(237 \pm 343 \mathrm{~s} ; \mathrm{p}<0.02$; fig. $2 \mathrm{a})$. Six $(35 \%)$ subjects achieved a $P$ th,max greater than their baseline result, $11(65 \%)$ subjects achieved a tlim greater than their baseline result and eight (47\%) subjects achieved a tlim of $15 \mathrm{~min}$.

ROC curves were used to determine the capacity of $P$ th,max and tlim to discriminate individuals who participated in H-IMT or S-IMT. The cut-off value with an optimal combination of sensitivity and specificity to distinguish the two groups was a $5.6 \%$ increase in $P$ th,max (sensitivity of $100 \%$ and specificity of $70.6 \%$ ) and a $44.3 \%$ increase in thim (sensitivity of $94 \%$ and specificity of $65 \%$ ). The area under the ROC curve for the percentage change in $P$ th, max $(0.87$; fig. $1 b)$ exceeded the area under the ROC curve for the percentage change in $t$ lim $(0.67$; fig. $2 b ; \mathrm{p}<0.05)$.

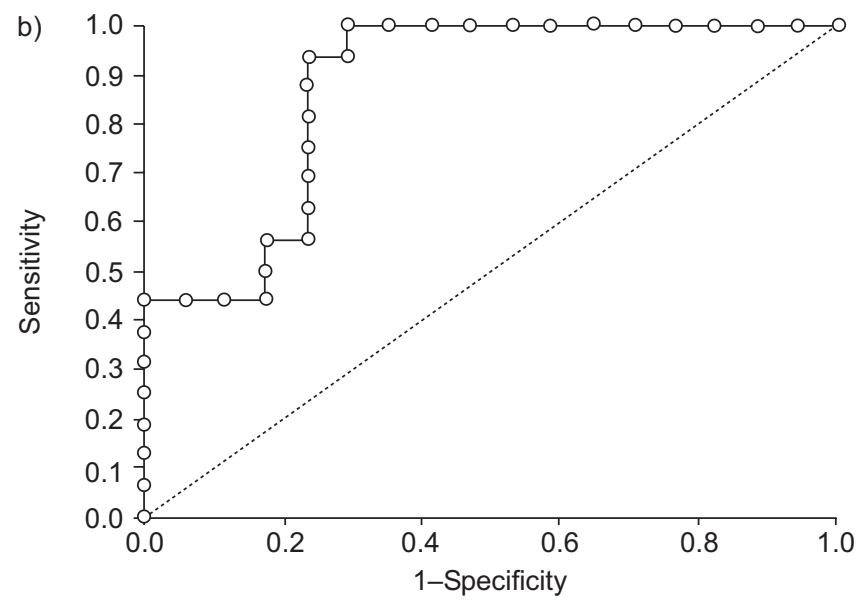

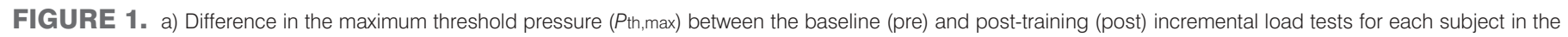

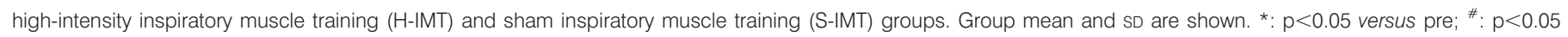
versus change following S-IMT. $1 \mathrm{cmH}_{2} \mathrm{O}=0.0978 \mathrm{kPa}$. b) Receiver operating characteristic curve for $P$ th,max. 

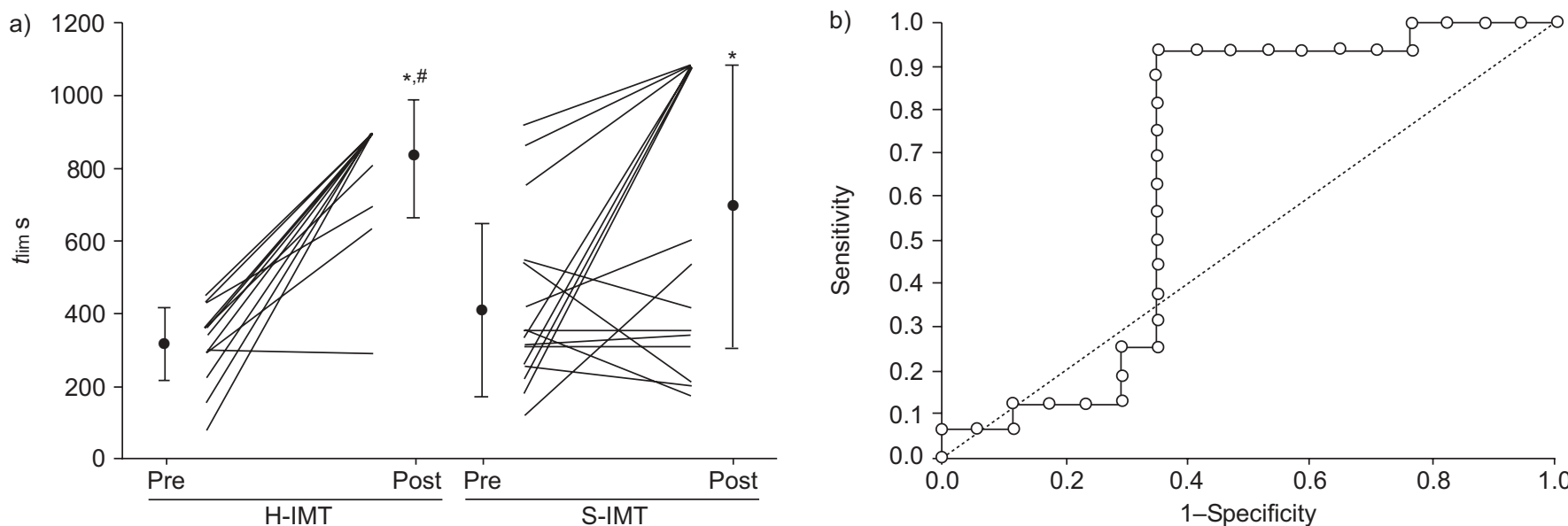

FIGURE 2. a) Difference in the time to exhaustion (tlim) between the baseline (pre) and post-training (post) constant load tests for each subject in the high-intensity inspiratory muscle training (H-IMT) and sham inspiratory muscle training (S-IMT) groups. Group mean and SD are shown. *: $p<0.05$ versus pre; ${ }^{\#}: p<0.05$ versus change following S-IMT. b) Receiver operating characteristic curve for tlim.

The increases in Pth,max and tlim following H-IMT were greater than any change observed in the S-IMT group $(\mathrm{p}<0.05$; figs $1 \mathrm{a}$ and 2a). The magnitude of change in each measure of inspiratory muscle function following H-IMT and S-IMT are shown in figure 3. Also displayed in figure 3 are the $95 \%$ confidence intervals associated with each change and the effect sizes for the difference in the magnitude of change in each measure between the groups.

\section{Effects of H-IMT and S-IMT on breathing pattern during loaded breathing tests}

Incremental load test

While minute ventilation at $P$ th, $\max$ was similar following H-IMT and S-IMT, differences in breathing pattern were
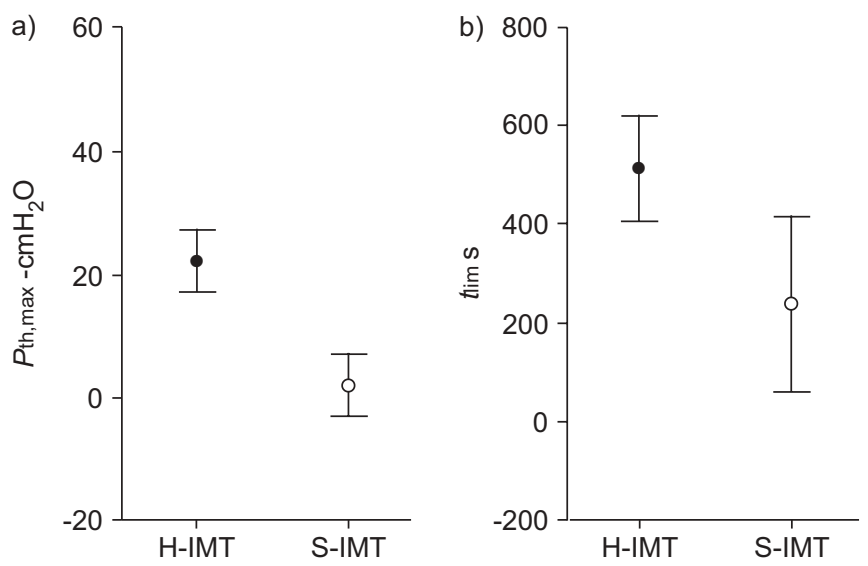

FIGURE 3. 95\% confidence intervals with effect sizes for the baseline to posttraining change in measurements of inspiratory muscle endurance. a) Maximum threshold pressure achieved during the incremental load test ( $P$ th,max; effect size 0.68), and b) time to exhaustion during the constant load test (tlim; effect size 0.44). - : high-intensity inspiratory muscle training ( $\mathrm{H}-\mathrm{IMT})$; $\bigcirc$ : sham inspiratory muscle training (S-IMT). $1 \mathrm{cmH}_{2} \mathrm{O}=0.0978 \mathrm{kPa}$. observed in both groups at $P$ th,max during the post-training test (table 1). Specifically, relative to the baseline test, during the post-training incremental loading test subjects breathed with a decreased inspiratory duty cycle, primarily as a consequence of increased expiratory time. Mean and peak inspiratory flow increased only in the H-IMT group. PTI was unchanged by training in either group.

\section{Constant load test}

Minute ventilation at thim during the constant load test was lower following S-IMT and unchanged following H-IMT (table 2). In general, the changes in breathing pattern observed during the incremental test were also noted during the constant load test. Specifically, inspiratory duty cycle decreased primarily as a consequence of an increase in expiratory time. As seen in the incremental load test, mean and peak inspiratory flow increased following H-IMT but not S-IMT. The changes in breathing pattern resulted in a reduction in PTI following H-IMT and S-IMT.

H-IMT versus S-IMT

While breathing pattern during the loaded breathing tests changed in both groups with training, comparison of the magnitude of change between the two groups revealed that during the incremental and constant load tests the increase in mean and peak inspiratory flow and the decrease in inspiratory duty cycle were significantly greater following H-IMT than S-IMT $(p<0.05)$. During the constant load test, the decrease in PTI following H-IMT was greater than any seen following S-IMT $(\mathrm{p}=0.001)$.

\section{DISCUSSION}

The present study is the first to compare the magnitude and specificity of change in measures of inspiratory muscle endurance assessed using an incremental and constant load test following a programme of H-IMT and S-IMT. Relative to any change seen following S-IMT, H-IMT was associated with significant increases in $P$ th, $\max$ and tlim. Therefore, both the incremental and constant load tests were able to discriminate 
TABLE 1 Breathing pattern variables collected during the pre- and post-training incremental load tests

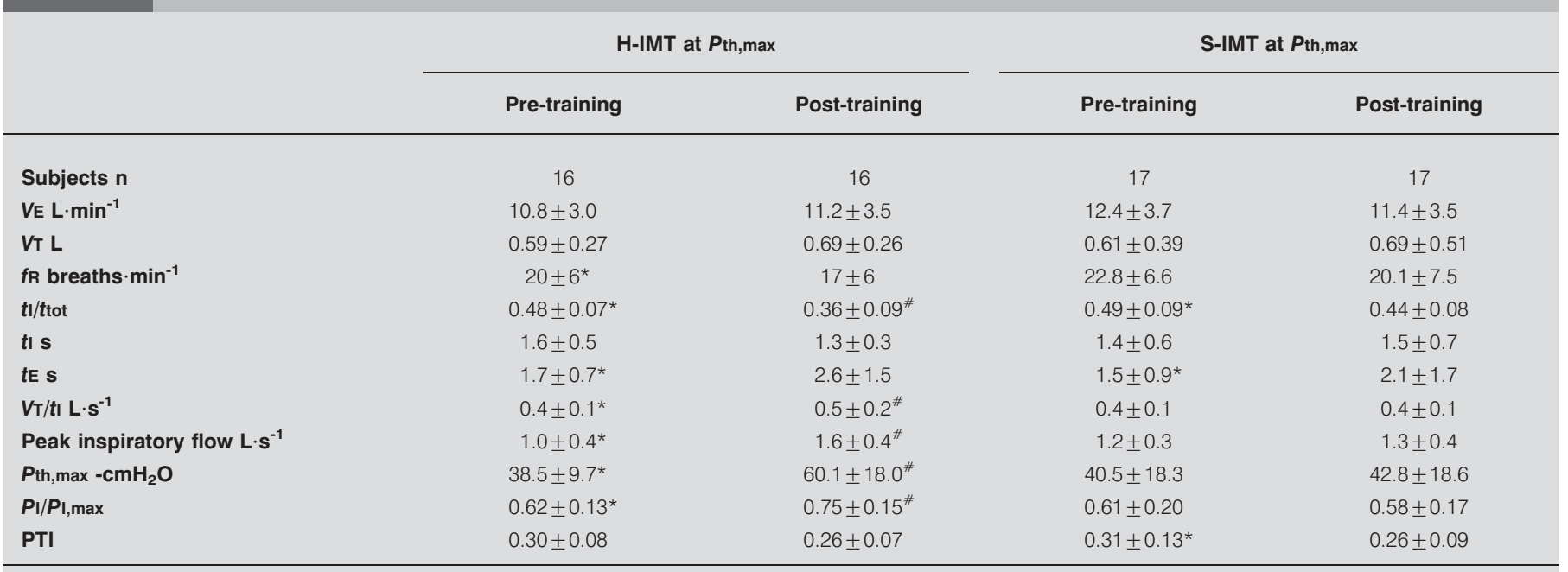

Data are presented as mean \pm SD, unless otherwise stated. H-IMT: high-intensity inspiratory muscle training; Pth,max: maximum inspiratory pressure achieved during the incremental load test; S-IMT: sham inspiratory muscle training; VE: minute ventilation; VT: inspiratory tidal volume; fR: respiratory frequency; tI/ttot: inspiratory duty cycle; $t I$ :

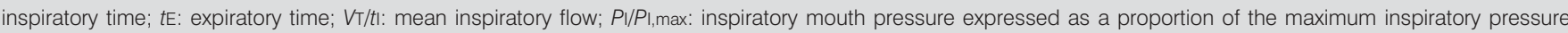
achieved during the same assessment session; PTI: pressure-time index. ${ }^{*}: p<0.05$ versus post-training measurements; ${ }^{*}$ : $p<0.05$ for magnitude of change following $\mathrm{H}-\mathrm{IMT}$ versus magnitude of change following S-IMT. $1 \mathrm{cmH}_{2} \mathrm{O}=0.0978 \mathrm{kPa}$.

between individuals who had participated in H-IMT from those who underwent S-IMT. It was notable, however, that the specificity and effect sizes for the magnitude of change in $P$ th,max were substantially greater than for tlim, suggesting that incremental load tests may more accurately discriminate a true training response.

\section{Inspiratory muscle training}

The magnitude of any physiological training response appears to be related to the magnitude of the training load $[6,10,26]$. For this reason, the subjects in the present study underwent H-IMT. Such a programme includes frequent rest periods, permitting relief of dyspnoea and local muscle fatigue, and thereby allowing greater loads to be achieved than would be possible with a continuous-based training protocol [16]. The current authors adopted such a protocol in order to optimise any potential improvement in inspiratory muscle endurance.

The magnitude of the increase in $P$ th, max and tlim was greater than reported in most previous studies of IMT in COPD [7, 9, $12,14,15]$. This most probably reflects the characteristics of the H-IMT programme, as high-intensity, interval-based, fully supervised whole-body exercise training performed over brief periods has been demonstrated to induce substantial increases in the endurance capacity of healthy subjects [27]. Indeed, the recent demonstration in COPD subjects of an increase in the proportion of type-I fibres in the external intercostal muscles in response to a 5-week, interval-based IMT programme performed at intensities considerably less than those achieved in the current study (40-50\% of PI,max) [14] reflects the rapidity of structural adaptations likely to occur with such programmes.

In contrast, S-IMT was performed at $10 \%$ of the baseline PI,max and was unchanged over the training period. This load was chosen to facilitate blinding of the subjects [28] without improving inspiratory muscle function [9]. By maximising the training load in one group (H-IMT) and selecting a load incapable of inducing physiological change in the other (S-IMT), the likelihood of inducing a detectable difference in inspiratory muscle function between groups following the training period was optimised.

\section{Incremental load test versus constant load test}

The 8-week programme of H-IMT resulted in significant increases in $P$ th,max and thim relative to measures obtained at baseline and to any change seen in the S-IMT group. These findings demonstrate that, with the sample size used in the present study $(n=33)$, both the incremental and constant load tests were able to discriminate between individuals who had participated in H-IMT from those who underwent S-IMT.

The 95\% confidence intervals and effect sizes for difference in magnitude of change in $P$ th, max and thim were calculated in order to quantify and compare the capacity of the incremental and constant load tests in order to discriminate between the H-IMT and S-IMT groups. The effect size for $P$ th,max $(0.68)$ is considered medium to large and the effect size for tim (0.44) is considered small to medium [29]. The large variability in the magnitude of change observed in tlim following S-IMT (fig. 3) is likely to have contributed to the smaller effect size. This finding is consistent with that of an earlier study [30], which demonstrated greater variability in the performance of a constant load test compared with an incremental load test when both were performed on multiple occasions.

The capacity of each measure to discriminate between individuals who had participated in H-IMT from those who underwent S-IMT was also evaluated in terms of their 


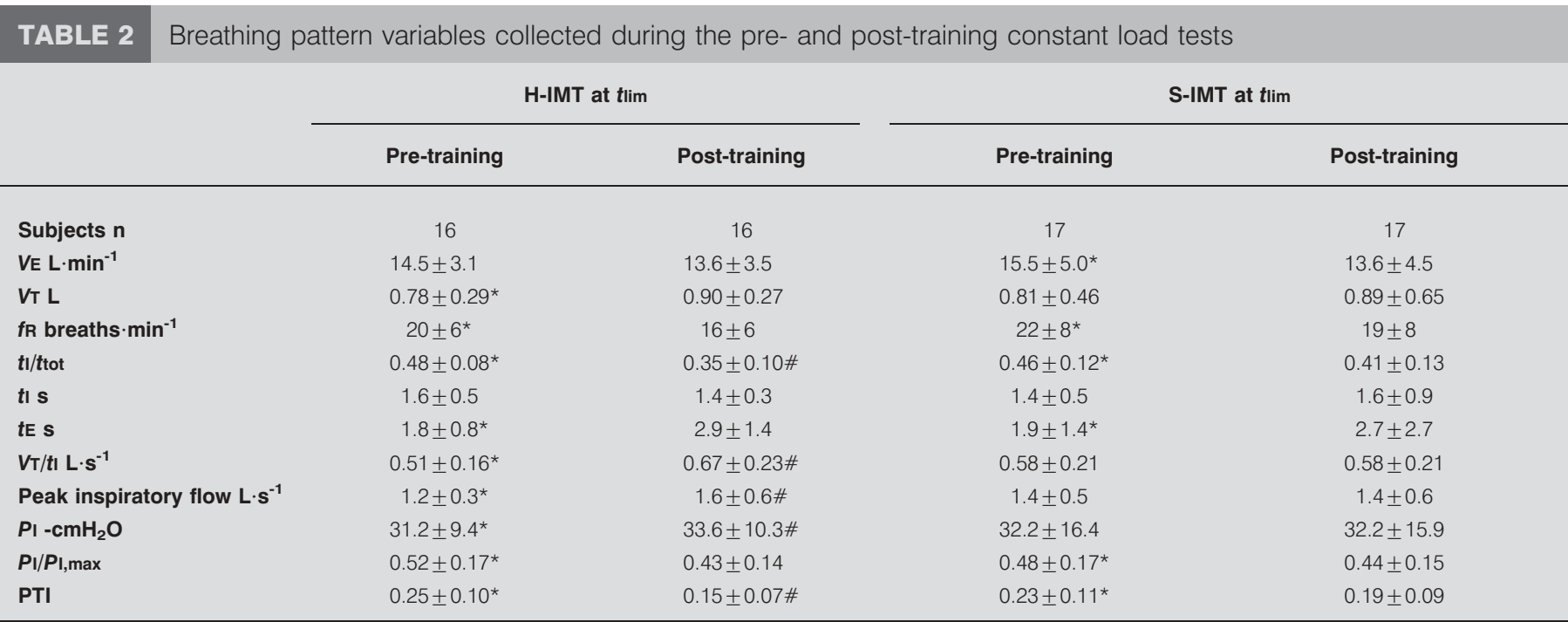

Data are presented as mean \pm SD, unless otherwise stated. H-IMT: high-intensity inspiratory muscle training; tlim: final minute of the constant load test; S-IMT: sham inspiratory muscle training; $V_{E}$ : minute ventilation; $V_{T}$ : inspiratory tidal volume; fR: respiratory frequency; $t / / t$ tot: inspiratory duty cycle; $t I$ : inspiratory time; $t E$ : expiratory time;

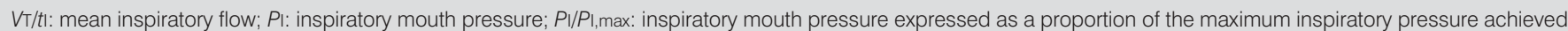
during the same assessment session; PTI: pressure-time index. *: $\mathrm{p}<0.05$ versus post-training measurements; ${ }^{*}: \mathrm{p}<0.05$ for magnitude of change following $\mathrm{H}-\mathrm{IMT}$ versus magnitude of change following S-IMT. $1 \mathrm{cmH}_{2} \mathrm{O}=0.0978 \mathrm{kPa}$.

sensitivity and specificity. These analyses showed that the change in measures obtained from both the incremental and constant load tests were equally sensitive in correctly identifying individuals who had undergone H-IMT. However, tlim was less specific in its capacity to distinguish individuals who had undergone H-IMT than $P$ th,max. This finding was reflected in the significantly greater area under the ROC curve for the change in $P$ th,max than the change in tlim. It is likely that the large number of subjects achieving a tlim of 15 min contributed to the reduced specificity and smaller area under the ROC for the constant load test. An alternative constant load test protocol designed to limit the number of subjects able to achieve a tlim of $15 \mathrm{~min}$ may have reduced the difference in the areas under the ROC curves between these two tests.

\section{Limitations}

A potential limitation in applying the constant load test to determine inspiratory muscle endurance was the requirement to terminate the test at $15 \mathrm{~min}$. By manipulating the inspiratory load, task failure (tlim) was able to be elicited in $<15 \mathrm{~min}$ in all subjects during the baseline test. However, following training, application of the same inspiratory load resulted in the test being terminated at $15 \mathrm{~min}$ in 75 and $47 \%$ of subjects in the H-IMT and S-IMT groups, respectively. Therefore, adjusting the load to elicit task failure within 5-10 min in the baseline constant load test resulted in failure to obtain a finite posttraining measurement of endurance in a large number of subjects. While increasing the initial load may have reduced the number of subjects who achieved 15 min during the posttraining test, doing so may well have decreased the initial tlim in many individuals to $<5 \mathrm{~min}$, reducing its value as an endurance measure [19]. Conversely, had subjects been permitted to continue past $15 \mathrm{~min}$, the magnitude of change in tlim between the two groups may have increased. However, a test of duration 20 or $30 \mathrm{~min}$, or longer, is likely to be of limited practical use in the clinical setting.

Another potential limitation of the study was the capacity of the subjects to adopt their own breathing pattern during the incremental and constant load tests. It is possible that the observed increases in $P$ th, max and tim reflect not only improved inspiratory muscle function, but also any training-related changes in breathing pattern. Indeed, previous studies have demonstrated that performance during assessments of inspiratory muscle endurance is influenced not only by the inspiratory load imposed [31] but also by the breathing pattern adopted [22, 32].

It was notable that, following training, the H-IMT group had increased mean and peak inspiratory flows during both the incremental and constant loading tests, whereas the S-IMT group did not. Such a strategy is an efficient way to deal with inspiratory threshold loads where, once the threshold pressure is exceeded, flow changes independently of pressure [20,33]. While these changes reflect greater muscle power output with increased velocity of muscle shortening during the inspiratory phase of the respiratory cycle [10, 34], the accompanying decrease in inspiratory duty cycle (and respiratory frequency) resulted in a decrease in the breath-by-breath load on the inspiratory muscles (i.e. PTI) in the case of the constant loading test, with no change in the case of the incremental loading test. These changes and their effects on inspiratory muscle work are consistent with a study by HART et al. [5], who showed an inverse linear relationship between inspiratory duty cycle and time to exhaustion while breathing against a constant inspiratory load. Hence, while an 8-week programme of H-IMT increased inspiratory muscle strength, power and 
velocity of shortening, the changes in breathing pattern adopted during the constant and incremental load tests served to minimise the overall load on the inspiratory muscles. Therefore, an improvement in Pth,max and tlim may not necessarily represent an improvement in muscle endurance when breathing pattern is unconstrained. While it is possible that the training-related increases in muscle strength and power were necessary pre-conditions for these changes in breathing pattern, these findings indicate that in order to be able to wholly attribute changes in $P$ th, max and tlim to improvements in inspiratory muscle function, breathing pattern during these tests should be controlled.

\section{Conclusion and recommendations}

Inspiratory muscle endurance measured either by $P$ th,max sustained during an incremental load test or tlim increased significantly following 8 weeks of H-IMT. Such changes are clinically relevant, as they are accompanied by a meaningful reduction in symptoms (dyspnoea during activities of daily living and fatigue) and a significant increase in 6-min walk distance [16]. The increases in both $P$ th,max and thim were greater following H-IMT following S-IMT. The effect size and specificity of the increase in $P$ th, max was substantially greater than those calculated for tlim. This was due, at least in part, to the substantial variability observed in the magnitude of change in thim following S-IMT. While these findings favour incremental over constant loading tests of inspiratory muscle endurance, it is possible that they are applicable only to tests of inspiratory muscle endurance in which individuals are free to adopt their own breathing pattern.

Marked changes in the breathing pattern adopted during each test were observed, particularly in the group who underwent high-intensity inspiratory muscle training. The magnitude of the effect of changes in breathing pattern was such that the overall load on the inspiratory muscles was either unchanged or decreased, even though high-intensity inspiratory muscle training resulted in increased inspiratory muscle strength, power and velocity of shortening. These data suggest that, in order to accurately interpret improved performance during incremental or constant load tests as an increase in respiratory muscle endurance, protocols should be adopted in which breathing pattern is controlled.

\section{ACKNOWLEDGEMENTS}

The authors gratefully acknowledge N. Cecins, D. Green, M. Phillips, T. van der Touw, G. Sturdy, R. Gupta, E. Gabbay, P. Winship, the Depts of Respiratory Medicine and Physiotherapy of Royal Perth Hospital (all Perth, Australia) and Sir Charles Gairdner Hospital (Nedlands, Australia) for their assistance.

\section{REFERENCES}

1 American Thoracic Society/European Respiratory Society. ATS/ERS statement on respiratory muscle testing. Am J Respir Crit Care Med 2002; 166: 518-624.

2 Lotters F, van Tol B, Kwakkel G, Gosselink R. Effects of controlled inspiratory muscle training in patients with COPD: a meta-analysis. Eur Respir J 2002; 20: 570-576.
3 Troosters T, Gosselink R, Decramer M. Respiratory muscle assessment. In: Gosselink R, Stam H, eds. Lung Function Testing. Eur Respir Mon 2005; 31: 57-71.

4 Martyn JB, Moreno RH, Pare PD, Pardy R. Measurement of inspiratory muscle performance with incremental threshold loading. Am Rev Respir Dis 1987; 135: 919-923.

5 Hart N, Hawkins P, Hamnegard CH, Green M, Moxham J, Polkey MI. A novel clinical test of respiratory muscle endurance. Eur Respir J 2002; 19: 232-239.

6 Larson JL, Kim MJ, Sharp JT, Larson DA. Inspiratory muscle training with a pressure threshold breathing device in patients with chronic obstructive pulmonary disease. Am Rev Respir Dis 1988; 138: 689-696.

7 Kim MJ, Larson JL, Covey MK, Vitalo CA, Alex CG, Patel M. Inspiratory muscle training in patients with chronic obstructive pulmonary disease. Nurs Res 1993; 42: 356-362.

8 Preusser BA, Winningham ML, Clanton TL. High- versus low-intensity inspiratory muscle interval training in patients with COPD. Chest 1994; 106: 110-117.

9 Heijdra YF, Dekhuijzen PNR, van Herwaarden CLA, Folgering HTM. Nocturnal saturation improves by targetflow inspiratory muscle training in patients with COPD. Am J Respir Crit Care Med 1996; 153: 260-265.

10 Villafranca C, Borzone G, Leiva A, Lisboa C. Effect of inspiratory muscle training with an intermediate load on inspiratory power output in COPD. Eur Respir J 1998; 11: 28-33.

11 Scherer TA, Spengler CM, Owassapian D, Imhof E, Boutellier U. Respiratory muscle endurance training in chronic obstructive lung disease. Am J Respir Crit Care Med 2000; 162: 1709-1714.

12 Sanchez Riera H, Montemayor Rubio T, Ortega Ruiz F, et al. Inspiratory muscle training in patients with COPD: effect on dyspnea, exercise performance, and quality of life. Chest 2001; 120: 748-756.

13 Hsiao SF, Wu YT, Wu HD, Wang TG. Comparison of effectiveness of pressure threshold and targeted resistance devices for inspiratory muscle training in patients with chronic obstructive pulmonary disease. J Formos Med Assoc 2003; 102: 240-245.

14 Ramirez-Sarmiento A, Orozco-Levi M, Guell R, et al. Inspiratory muscle training in patients with chronic obstructive pulmonary disease: structural adaptation and physiologic outcomes. Am J Respir Crit Care Med 2002; 166: 1491-1497.

15 Weiner P, Magadle R, Beckerman M, Weiner M, BerarYanay N. Comparison of specific expiratory, inspiratory, and combined muscle training programs in COPD. Chest 2003; 124: 1357-1364

16 Hill K, Jenkins SC, Philippe D, et al. High-intensity inspiratory muscle training in COPD. Eur Respir J 2006; 27: 1119-1128.

17 Hankinson JL, Odencrantz JR, Fedan KB. Spirometric reference values from a sample of the general U.S. population. Am J Respir Crit Care Med 1999; 159: 179-187.

18 Sturdy GA, Hillman DR, Green DJ, Jenkins SC, Cecins NM, Eastwood PR. The effect of learning on ventilatory responses to inspiratory threshold loading in COPD. Respir Med 2004; 98: 1-8. 
19 Nickerson BG, Keens TG. Measuring ventilatory muscle endurance in humans as sustainable inspiratory pressure. $J$ Appl Physiol 1982; 52: 768-772.

20 Eastwood PR, Hillman DR. A threshold loading device for testing of inspiratory muscle performance. Eur Respir J 1995; 8: 463-466.

21 Flynn MG, Barter CE, Nosworthy JC, Pretto JJ, Rochford PD, Pierce RJ. Threshold pressure training, breathing pattern, and exercise performance in chronic airflow obstruction. Chest 1989; 95: 535-540.

22 Bellemare F, Grassino A. Effect of pressure and timing of contraction on human diaphragm fatigue. J Appl Physiol 1982; 53: 1190-1195.

23 Rochester DF. Effects of COPD on the respiratory muscles. In: Cherniack NS, ed. Chronic Obstructive Pulmonary Disease. Philadelphia, W.B. Saunders and Company, 1991; pp. 134-157.

24 Goldstein RS, De Rosie J, Long S, Dolmage T, Avendano MA. Applicability of a threshold device for inspiratory muscle testing and training in patients with COPD. Chest 1989; 96: 564-571.

25 Sturdy GAN, Hillman DR, Green DJ, Jenkins SC, Cecins N, Eastwood PR. Feasibility of high-intensity, interval-based respiratory muscle training in COPD. Chest 2003; 123: 142-150.

26 Belman MJ, Shadmehr R. Targeted resistive ventilatory muscle training in chronic obstructive pulmonary disease. J Appl Physiol 1988; 65: 2726-2735.
27 Burgomaster KA, Hughes SC, Heigenhauster GJ, Bradwell SN, Gibala MJ. Six sessions of sprint interval training increases muscle oxidative potential and cycle endurance capacity in humans. J Appl Physiol 2005; 98: 1985-1990.

28 Lisboa C, Villafranca C, Leiva A, Cruz E, Pertuze J, Borzone $\mathrm{G}$. Inspiratory muscle training in chronic airflow limitation: effect on exercise performance. Eur Respir J 1997; 10: 537-542.

29 Cohen J. Statistical Power Analysis for the Behavioural Sciences. 2nd Edn. Hillsdale, Lawrence Earlbaum Associates, 1988.

30 McElvaney G, Fairbarn MS, Wilcox PG, Pardy RL. Comparison of two-minute incremental threshold loading and maximal loading as measures of respiratory muscle endurance. Chest 1989; 96: 557-563.

31 Roussos CS, Macklem PT. Diaphragmatic fatigue in man. J Appl Physiol 1977; 43: 189-197.

32 Clanton TL, Dixon GF, Drake J, Gadek JE. Effects of breathing pattern in inspiratory muscle endurance in humans. J Appl Physiol 1985; 59: 1834-1841.

33 Eastwood PR, Hillman DR, Morton AR, Finucane KE. The effects of learning on the ventilatory responses to inspiratory threshold loading. Am J Respir Crit Care Med 1998; 158: 1190-1196.

34 Lisboa C, Munoz V, Beroiza T, Leiva A, Cruz E. Inspiratory muscle training in chronic airflow limitation: comparison of two different training loads with a threshold device. Eur Respir J 1994; 7: 1266-1274. 